\title{
Trends of Rotavirus in Nepal
}

\author{
Pandey BD, Pun SB
}

Sukraraj Tropical and Infectious Disease Hospital, Teku, Kathmandu, Nepal.

Everest International Clinic and Research Center, Kathmandu, Nepal

\section{Corresponding Author}

Basu Dev Pandey

Sukraraj Tropical and Infectious Disease Hospital, Kathmandu, Nepal.

Email: basupandey@wlink.com.np

\section{Citation}

Pandey BD, Pun SB. Trends of Rotavirus in Nepal. Kathmandu Univ Med J 2011;33(1)32-5.

\begin{abstract}
Background

Rotavirus gastroenteritis is the major cause of morbidity and mortality in infants and young children worldwide. Safe and effective rotavirus vaccine is needed to have significant impact on severe rotavirus gastroenteritis. Currently, two live oral rotavirus vaccines have been licensed in many countries. Knowledge on distribution of human rotavirus of $G$ and $P$ types are essential before rotavirus vaccines are introduced in the target populations.
\end{abstract}

\section{Objective}

To observe the trends of rotavirus strains in children below five years of age, during the years, 2003-2005 in Nepal.

\section{Methods}

Stool specimen collected from children with acute diarrhea who were referred to observation unit or hospitalized in Kanti Children's Hospital between 2003 and 2005 were examined. Meteorological data was obtained from Ministry of Environment, Nepal to examine the possibility on the impact of weather on rotavirus infection.

\section{Results}

Of 1250 stool specimens, $271(22 \%)$ were positive for rotavirus by Rotaclone ELISA. G1 was the most common serotype in the first year of study, and G2 in the following year. G12 serotype emerged and remained predominant in two consecutive years. In addition, G9 and G3 emerged in the second year of the study. Children less than three years of age were commonly affected. The records reveal that rotavirus infection is related to the climate, and is commonly seen in the dry season, peaking in January.

\section{Conclusions}

Continued surveillance of different regions is needed to monitor the trend of rotavirus strains and to establish rotavirus disease burden, which will help policy makers to make a decision in introducing rotavirus vaccine in Nepal.

\section{KEY WORD}

Nepal, rotavirus, serotype

\section{INTRODUCTION}

Rotavirus is a major cause of acute gastroenteritis (AGE) in children under five years of age worldwide. It is estimated that 702,000 children deaths occurs due to rotavirus gastroenteritis annually, the majority occurring in developing countries. ${ }^{1}$ Currently two live, oral, attenuated rotavirus vaccines have been developed and are now introduced in childhood immunization schedules in more than 100 countries. $^{2}$
Rotavirus, a genus within the Reoviridae family, has 11 segments of double stranded RNA as its genome. Segment nine and four code for VP7 and VP4 proteins respectively and are the major neutralization antigens. VP7 defines the $\mathrm{G}$ (glycoprotein) and VP4 defines the P (protease -sensitive) genotypes. ${ }^{3}$ Currently $15 \mathrm{G}$ and $26 \mathrm{P}$ genotypes have been found in humans and animals. ${ }^{4}$ Globally G1, G2, G3, and $\mathrm{G} 4$ are the most common $\mathrm{G}$ genotypes and commonly combined with P [8], P [4] and P [6]. ${ }^{5}$ Uncommon serotypes $\mathrm{G} 5, \mathrm{G} 8, \mathrm{G} 10, \mathrm{G} 11$, and $\mathrm{G} 12$ were reported from various parts of the world. ${ }^{6-10}$ 
Nepal is a resource-poor, sub-tropical and landlocked country in South Asia, bordering the People's Republic of China to the north and India to the east, west and south. Nepal has four seasons: spring (March-May), summer (June-August), autumn (September-November) and winter (December-February). In addition, there are rainy monsoon season (June-September) and dry season (October-May). Mortality rate among children less than five years of age remains high in Nepal, and diarrheal disease is a major contributor. ${ }^{11}$ Among diarrheal diseases, rotavirus is one of the most common causes of non bacterial gastroenteritis in children. ${ }^{12-16}$ Although, several studies have been done on rotavirus gastroenteritis in Nepal, molecular characterizations of rotavirus gastroenteritis have only been initiated since 2003. ${ }^{17}$ Two rotavirus vaccines (Rotateq and Rotarix) are now made available in the global market.

In anticipation of rotavirus vaccine in the routine childhood immunization schedule in Nepal, the present study attempts to observe the trend of rotavirus strains circulating in children less than five years of age between 2003 and 2005. This information will provide the background to the evaluation of vaccine effectiveness after the introduction of rotavirus vaccine in Nepal.

\section{METHODS}

Stool specimen were collected from children less than five years of age with acute diarrhea who were referred to observation unit or hospitalized in Kanti Children's Hospital $(\mathrm{KCH})$ between 2003 and 2005. They were examined in order to identify the distribution of rotavirus $\mathrm{G}$ serotypes. A commercially available enzyme-linked immunosorbent assay (ELISA) kit (Rotaclone; Meridian Bioscience Inc., Cincinnati, $\mathrm{OH}$ ) was used to detect group $A$ rotavirus antigen. $\mathrm{G}$ types were determined by reverse transcriptionPCR (RT-PCR) using a method described previously ${ }^{18}$ and non-typeable samples were further characterized by sequencing. ${ }^{17,19}$ Meteorological data was obtained from Department of Science and Technology, Department of Hydrology and Meteorology, Ministry of Environment, Nepal to examine the impact of weather on rotavirus infection. Statistic analysis was performed using Epi Info (Version 3.5.1) software package when appropriate and $P$ values of $<0.05$ were defined as statistically significant.

\section{RESULTS}

The present study was carried out between 2003 and 2005 at $\mathrm{KCH}$, Kathmandu. Among stool samples taken from children less than five years of age with AGE, 22\% (271/1250) were positive by ELISA method. Of 666 samples, 116(17\%) were positive for rotavirus in 2003-2004, whereas $155(27 \%)$ out of 584 samples in 2004-2005.

In 2003-2004, molecular study was initiated, where the most commonly detected serotype was G1 (77\%) followed by G12 (20\%) $)^{17}$ (Fig.1). In contrast, G2 (39\%) was the most

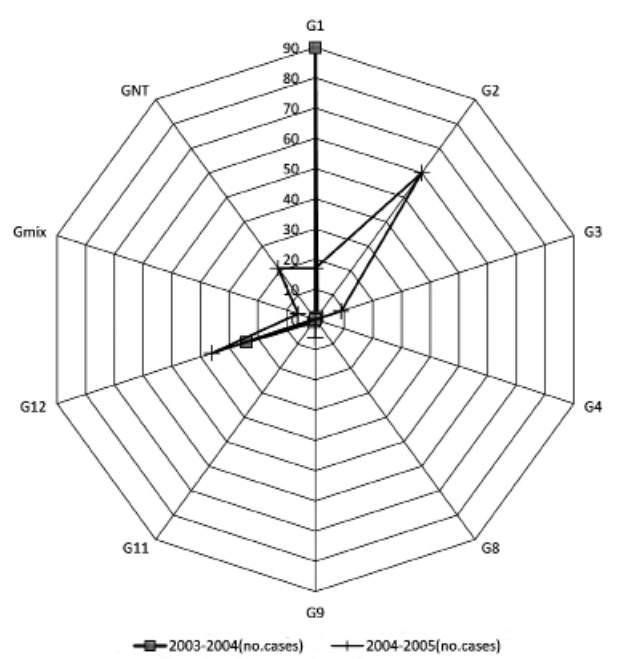

Figure 1. Distribution of rotavirus G serotypes among children, betweSeptember 2003 and July 2005 in Kanti Children's Hospital Kathmandu, Nepal.

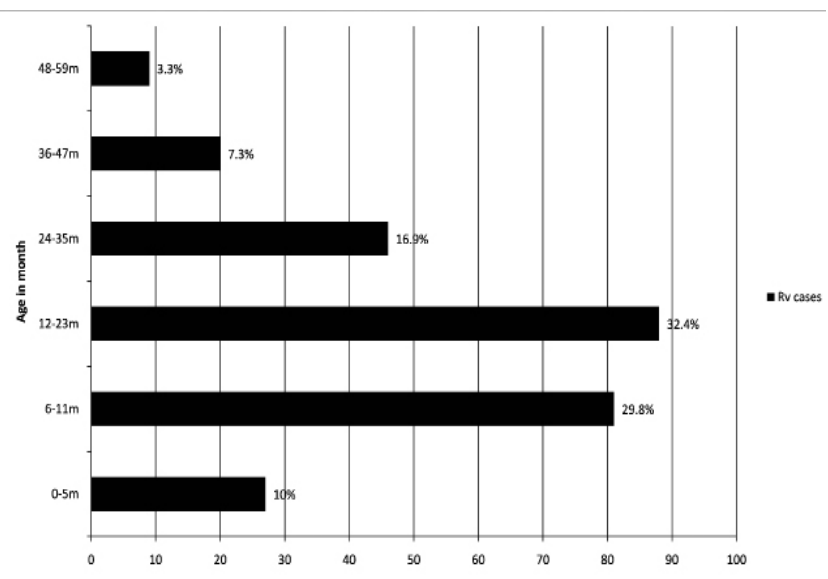

Figure 2. Age distribution of rotavirus infection among children, between September 2003 and July 2005 in Kanti Children's Hospital, Kathmandu, Nepal.

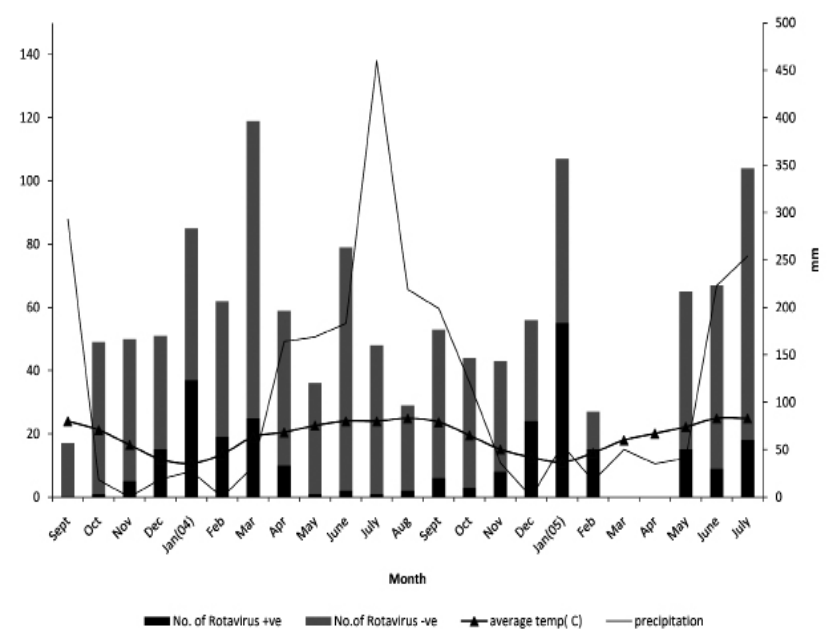

Figure 3. Seasonal trend of rotavirus detection in children less than 5 years, between September 2003 and July 2005 in Kanti Children's Hospital, Kathmandu, Nepal. 
commonly identified VP7 serotype followed by G12 (23\%) in 2004-2005. ${ }^{19}$ In this regard G12, an unusual serotype, was found to be emerged and stable in two consecutive years. G12, an unusual serotype was emerging during two consecutive years: 2003-2004 (Fig. 1) and 2004-2005.

Rotavirus was detected in all age groups in children. However, more than $85 \%$ of rotavirus cases were detected among children in less than three years of age (Fig.2).

In order to clarify the impact of climate on rotavirus infection in Nepal, we examined the monthly detection of rotavirus infection and compared them with average temperature and rainfall. Although rotavirus was detected throughout the year, there was a seasonal variation in occurrence of rotavirus infection, peaking in January (Fig. 3). Rotavirus infection mostly occurred during the cool and dry season from December to February.

\section{DISCUSSION}

Diarrhea among children less than five years of age is one of the major public health problems in Nepal. Physicians often do not seek pathogens responsible for the diarrhea because it does not change the treatment plan. Among diarrheal pathogens, rotaviruses have been identified as the most frequent causative agent in young children. Very few studies have been done to determine rotavirus infections, in patients with acute diarrhea in Nepal. While the detection of rotavirus infection using molecular technique has not been described before 2003.

In our study, positivity for rotavirus was found in $17 \%$ in 2003- 2004 and in 27\% in 2004-2005. This is in agreement with most of previous studies from Nepal, although rotavirus positivity rate was shown as high as $39 \% .{ }^{14}$ Differences in positivity rate among studies may be attributable to study period, places and study design.

G12, an unusual serotype was emerging during two consecutive years: 2003-2004 (Fig.1) and 2004-2005. Similar observations were made in India, where $\mathrm{G} 12$ has been continuously reported since $2000 .{ }^{20}$ Interestingly, Nepali G12 have been found associated with P [8], P [6] and P [4] VP4 P genotypes (data not shown).

Whole genome analysis of G12 strains circulating in Nepal could shed light on their origin and evolution. To our surprise, G11 was detected in the year 2003-2004 ${ }^{17}$; it was, however, not detected in the following year. Currently, there has been reported G11 from Bangladesh. ${ }^{9}$ Nepalese G11 has shown close genetic relationship with Bangladeshi G11 strains, when compared to major antigenic regions. ${ }^{17}$ G11 found in Nepal could be introduced from Bangladesh and reassorted with locally circulating strains. On the other hand, it is also possible that G11 was first introduced in Nepal due to nature of VP7 gene as porcine origin, where pig farming is common, but uncommon in Bangladesh. ${ }^{21}$ Close geographical location between Nepal and Bangladesh could facilitate the transmission of G11 strain during people's movement, could be a possible explanation for detecting G11 strain in both countries concurrently. In addition, G3 and G9 have been emerged in the second year of study, which did not appear in the preceding year, suggesting that these serotypes might have emerging in Nepal. Nevertheless, it is important to continue rotavirus surveillance to support or refute this hypothesis.

Rotavirus was detected in all age groups in children. However, more than $85 \%$ of rotavirus cases were detected among children in less than three years of age (Fig.2). Previous studies done in Nepal have shown similar findings. ${ }^{15,16}$ However, this was in contrast to other studies done in India, Pakistan and Bangladesh, where rotavirus had been mostly occurred (>90\%) during the first two years of life. ${ }^{22,23}$ Nevertheless, age group wise, in 12-23 months, rotavirus detection rate remained the highest (32.4\%) while 48-59 months age group was the lowest $(3.3 \%)(p<0.001)$.

We found rotavirus infection to occur mostly during the cool and dry season from December to February, peaking in January. This finding was in agreement with those reported earlier in Nepal. ${ }^{15,16}$ In rainy monsoon and summer season (June to September), rotavirus infection was rarely detected. In contrast, in Bangladesh, rotavirus infection was also observed to peak during the monsoon. It was hypothesized that the increase in water level of rivers resulted in an increased chance of fecal contamination of water. ${ }^{9}$ However, the frequency of rotavirus associated diarrhea in children during rainy monsoon season was not determined.

\section{CONCLUSION}

Rotavirus diarrhea is a serious health problem and life threatening that affects infants and young children in developing countries. ${ }^{1}$ Improved hygiene conditions alone are not enough to decrease the incidence of rotavirus associated diarrhea significantly. The implementation of an effective vaccine against rotavirus diarrhea would be the best option to address this problem. Currently available rotavirus vaccines have shown high efficacy in clinical trials and are undergoing clinical trials in several countries across Africa and Asia in order to determine their effectiveness. ${ }^{4}$

Recently the World Health Organization has recommended global use of rotavirus vaccines. Hence, in anticipating of rotavirus vaccine in Nepal, this study could provide the key to understand the trend of rotavirus strains in children less than five years of age, which will be important for evaluating the result of vaccine effectiveness in the background of diverse strains after the introduction of rotavirus vaccine.

\section{ACKNOWLEDGMENT}

The Authors wish to thank Prof Osamu Nakagomi 


\section{REFERENCES}

1. Cunliffe NA, Nakagomi O. A critical time for rotavirus vaccines: a review. Expert Rev. Vaccines. 2005; 4: 521-32.

2. Nakagomi O, Cunliffe NA. Rotavirus vaccines: entering a new stage of deployment. Curr Opin Infect Dis 2007;20: 501-07

3. Estes MK, Kapikian AZ. Rotaviruses. In: Knipe DM, Howley PM, Griffin $\mathrm{DE}$, Lamb RA, Martine MA, Roizman B, Straus SE (ed.). Fields virology, $5^{\text {th }}$ ed.Lippicott, Williams, Wilkins, Philadelphia; 2007. P. 1917-74.

4. Cunliffe NA, Nakagomi O. Introduction of rotavirus vaccines in developing countries: remaining challenges. Annals of Tropical Pediatrics. 2007; 27: 157-67.

5. Kaga $E$, Lizuka M, Nakagomi T, Nakagomi O. The distribution of $G$ (VP7) and P (VP4) Serotypes among Human Rotaviruses Recovered from Japanese Children with Diarrhea. Microbiol Immunol. 1994; 38 : 317-20

6. Cunliffe NA, Gondwe JS, Broadhead RL, Molyneux ME, Woods PA, Bresee JS et al. Rotavirus $G$ and $P$ types in children with acute diarrhea in Blantyre , Malawi, from 1997 to 1998: Predominance of novel P [6] G8 strains. J Med Virol. 1999; 57: 308-12.

7. Santos N, Hoshino Y. Global distribution of rotavirus serotypes/ genotypes and its implication for the development and implementation of an effective rotavirus vaccine. Rev Med Virol. 2005; 15: 29-56

8. Ahmed K, Anh DD, Nakagomi O. Rotavirus G5 P [6] in child with Diarrhea, Vietnam. Emerg Infect Dis. 2007; 13: 1232-35.

9. Rahman M, Sultana R, Ahmed G, Nahar S, Hassan ZM, Saiada F, et al. Prevalence of G2 P [4] and G12 P [6] Rotavirus, Bangladesh. Emerg Infect Dis. 2007; 13: 18-24.

10. Cunliffe NA, Ngwira BM, Dove W, Nakagomi O, Nakagomi T, Perez A, et al. Serotype G12 Rotaviruses, Lilongwe, Malawi. Emerg Infect Dis. 2009; 15: 87-90.

11. Human Development Report, Nepal. United Nation Development Project (UNDP). 2007-2008

12. Sherchand JB, Larsson S, Rana BJ, Dixit H, Bam DS, Adhikari RK, et al. On the incidence of rotavirus and enteric adenovirus diarrhoea in children attending the outpatient department of Kanti Children's Hospital and general practitioners in the Kathmandu area. J. Nepalese Med. Assoc. 1992; 30:149-53.
13. Ono K, Rai SK, Chikahira FT, Shibata H, Wada Y, Tsuji H, et al. Seasonal distribution of enteropathogens detected from diarrheal stools and water samples collected in Kathmandu, Nepal. Southeast Asian J Trop Med Public Health. 2001; 32: 520-26.

14. Shariff M, Deb M, Singh R. A study of diarrhea among children in Eastern Nepal with special reference to rotavirus. Indian J Med Microbiol. 2003; 21: 87-90.

15. Sherchand JB, Haruki K. Rotavirus Diarrhea in Children and Animal of Urban and Rural Nepal. J Nepal Health Res Counc. 2004; 2: 5-8.

16. Maharjan A, Sherchand JB, Pradhan B, Paudyal A, Panta AR. Rotavirus Infection among Diarrheal Children Attending Kanti Children Hospital, Kathmandu, Nepal. J Nepal Health Res Counc. 2007; 4: 34-40.

17. Uchida R, Pandey BD, Sherchand JB, Ahmed K, Yokoo M, Nakagomi T, et al. Molecular epidemiology of rotavirus diarrhea among children and adults in Nepal: Detection of G12 strains with P [6] or P [8] and a G11 P [25] Strain. J Clin Microbiol. 2006; 44: 3499-505

18. Gouvea V, Glass RI, Wood P, Taniguchi K, Clark HF, Forreter B et al. Polymerase chain reaction amplification and typing of rotavirus nucleic acid from stool specimens. J Clin Microbiol. 1990; 28: 276-82

19. Pun SB, Nakagomi T, Sherchand JB, Pandey BD, Cuevas LE, Hart CA, et al. Detection of G12 human rotaviruses in Nepal. Emerg Infect Dis. 2007; 13: 482-84.

20. Sharma S, Ray P, Gentsch JR, Glass RI, Kalra V, Bhan MK. Emergence of G12 Rotavirus Strains in Delhi, India, in 2000 to 2007. J Clin Microbiol. 2008; 46: 1343-48.

21. Rahman M, Matthijnssens J, Nahar S, Podder G, Sack DA, Azim T, et al. Characterization of a Novel P [25], G11 Human Group A Rotavirus. J Clin Microbiol. 2005;43: 3208-12

22. Bahl R, Ray P, Subodh S, Shambhaskar P, Saxena M, Prashar U,et al. Incidence of Severe Rotavirus Diarrhea in New Delhi, India, and G and P Types of the Infecting Rotavirus Strains. J Infect Dis. 2005; 192 (Suppl 1) 114-19.

23. Nishio O, Matsui K, Oka T, Ushijima H, Mubina A, Dure-Samin A, et al. Rotavirus Infection among infants with diarrhea in Pakistan. Pediatric International. 2000; 42: 425-27. 